\title{
Monte Carlo simulation of Touschek effect
}

\author{
Aimin Xiao* and Michael Borland \\ Accelerator Systems Division, Advanced Photon Source, Argonne National Laboratory, 9700 South Cass Avenue, \\ Argonne, Illinois 60439, USA
}

(Received 29 April 2009; revised manuscript received 15 June 2010; published 30 July 2010)

\begin{abstract}
We present a Monte Carlo method implementation in the code ELEGANT for simulating Touschek scattering effects in a linac beam. The local scattering rate and the distribution of scattered electrons can be obtained from the code either for a Gaussian-distributed beam or for a general beam whose distribution function is given. In addition, scattered electrons can be tracked through the beam line and the local beamloss rate and beam halo information recorded.
\end{abstract}

DOI: 10.1103/PhysRevSTAB.13.074201

PACS numbers: 29.20.-c, 29.27.-a, 41.75.-i

\section{INTRODUCTION}

Touschek scattering results from a Coulomb collision of two relativistic electrons in a particle beam, producing an instantaneous change in particle energy. Because of relativistic effects, the amount of momentum transferred from the transverse to the longitudinal plane in the rest frame is increased by the Lorentz factor $\gamma$ in the lab frame. If the resulting energy deviation is outside the radio-frequency acceptance, or the resulting closed orbit or betatron oscillation exceeds dynamic or physical aperture limitations, the electron is lost.

The Touschek effect was first found and studied experimentally in the low-energy electron storage ring ADA [1] and several theoretical investigations of the effect have been made [2,3]. It is one of the most important beam lifetime limiting effects in state-of-the-art low-emittance electron storage rings.

Particle loss due to Touschek scattering has been largely ignored for single-pass accelerators (e.g., linacs and transport lines) in the past. In such systems, the beam only passes through once and the product of the transit time and Touschek scattering rate is generally very small. However, this thinking has changed given the recent interest in light sources based on energy recovery linacs (ERLs) [4]. These proposed sources feature an intense electron bunch with ultralow transverse emittance and a very short bunch length, which combine to cause a much higher Touschek scattering rate. This, coupled with the high bunch repetition rate that is required to provide high average brightness, means that the total particle loss rate is no longer something we can ignore.

This is a concern for a possible ERL upgrade of the Advanced Photon Source (APS), since the existing radiation shielding is not designed for high continuous loss rates [5]. A preliminary theoretical analysis has been performed previously using Piwinski's formula [6]. To obtain detailed information about the scattered particle distribution and

*xiaoam@aps.anl.gov determine particle loss rates and positions, we developed a Monte Carlo simulation of Touschek scattering as part of the code ELEGANT [7].

In this paper, we start by reviewing the theory of the Touschek effect, then describe the method used for Monte Carlo simulation. This method is applied to an example lattice [8] and the calculated Touschek scattering rates for a Gaussian-distributed beam are compared with Piwinski's formula results. To make our simulation more general, this method is then extended to allow a general beam distribution. The calculation of beam-loss rate and location is explained with an emphasis on efficient simulation. Finally, we present an application of this method to a proposed APS-ERL lattice design.

\section{THEORY DESCRIPTION}

Before describing the simulation technique, we begin with a review of the general theory of Touschek scattering. The theory involves quantities in both the laboratory coordinate system and the center-of-mass (c.m.) systems. For clarity, we use an asterisk $(*)$ to denote all quantities in the c.m. system. Quantities without this symbol refer to the laboratory coordinate system.

In the c.m. system, the probability of one of the two interacting electrons being scattered into a solid angle $d \Omega^{*}$ is given by the differential Møller cross section [9],

$$
\begin{aligned}
\frac{d \sigma^{*}}{d \Omega^{*}}\left(\Theta^{*}, \Psi^{*}\right)= & \frac{r_{e}^{2}}{4 \gamma^{* 2}}\left[\left(1+\frac{1}{\beta^{* 2}}\right)^{2} \frac{4-3 \sin ^{2} \Theta^{*}}{\sin ^{4} \Theta^{*}}\right. \\
& \left.+\frac{4}{\sin ^{2} \Theta^{*}}+1\right],
\end{aligned}
$$

where $r_{e}$ is the classical electron radius; $\gamma^{*}$ and $\beta^{*}$ are the relative energy and velocity of the scattered electron in the c.m. system, respectively; $\Theta^{*}$ is the angle between the momentum before and after scattering; and $d \Omega^{*}=$ $\sin \Theta^{*} d \Theta^{*} d \Psi^{*}$. Figure 1 illustrates the process. We chose the coordinate system with $x^{*}$ axis collinear with the c.m. momentum vectors of the incident particles, and $z^{*}$ the direction of Lorentz transformation. 


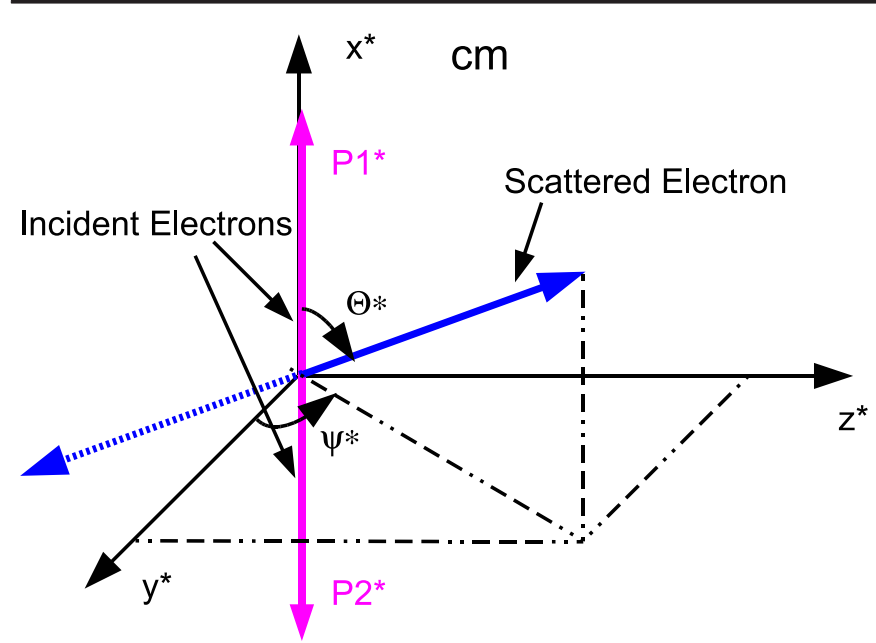

FIG. 1. (Color) Electron momenta before (magenta) and after (blue) scattering.

The total scattering rate $R$ is given by the integral over all possible scattering angles and over all electrons in the bunch. In the c.m. system,

$$
R^{*}=2 \int\left|v^{*}\right| \sigma^{*} \rho\left(\vec{x}_{1}^{*}\right)^{*} \rho\left(\vec{x}_{2}^{*}\right)^{*} d V^{*}
$$

where $v^{*}$ is the scattered electrons' velocity, $\vec{x}^{*}=$ $\left(x^{*}, y^{*}, z^{*}, p_{x}^{*}, p_{y}^{*}, p_{z}^{*}\right), \rho\left(\vec{x}_{i}^{*}\right)^{*}$ is the electron phase-space density, $\quad d V^{*}=d x^{*} d y^{*} d z^{*} d p_{x 1}^{*} d p_{y 1}^{*} d p_{z 1}^{*} d p_{x 2}^{*} d p_{y 2}^{*} d p_{z 2}^{*}$, and $\sigma^{*}$ is the total Møller cross section, which results from the integral of the differential cross section over the solid angle $d \Omega^{*}$ with $\Theta^{*} \in\left(0, \frac{\pi}{2}\right], \Psi^{*} \in[0,2 \pi]$ :

$$
\sigma^{*}=\int_{0}^{2 \pi} \int_{0}^{\pi / 2} \frac{d \sigma^{*}}{d \Omega^{*}} \sin \Theta^{*} d \Theta^{*} d \Psi^{*}
$$

The reason for restricting the integral to $\Theta^{*} \in\left(0, \frac{\pi}{2}\right]$ is that, if one electron is scattered into the region $0<\Theta^{*} \leq$ $\frac{\pi}{2}$, then the other is scattered into the region $\frac{\pi}{2} \leq \Theta^{*}<\pi$. The factor " 2 " in Eq. (2) includes both regions.

Transforming to the laboratory coordinate system gives

$$
|v| \sigma=\frac{\left|v^{*}\right|}{\gamma} \frac{\sigma^{*}}{\gamma}
$$

and

$$
R=2 \int|v| \sigma \rho\left(\vec{x}_{1}\right) \rho\left(\vec{x}_{2}\right) d V
$$

with

$$
d V=d x_{\beta} d y_{\beta} d \Delta z d x_{\beta 1}^{\prime} d x_{\beta 2}^{\prime} d y_{\beta 1}^{\prime} d y_{\beta 2}^{\prime} d \Delta p_{1} d \Delta p_{2} .
$$

Piwinski [3] made a detailed evaluation of Eq. (5) for a Gaussian-distributed bunch and derived a general formula [Equation (41) in [3]] for the Touschek lifetime. We will use the symbol $R_{\mathrm{P}}$ to denote the Touschek scattering rate from Piwinski's formula.

\section{MONTE CARLO SIMULATION}

The integral of Eq. (5) can be computed using the Monte Carlo integration technique with $N$ uniformly distributed random points in the $n$-dimensional volume $V$, e.g.,

$$
\int_{V} f(\vec{x}) d \vec{x} \approx \frac{V}{N} \sum_{i=1}^{N} f\left(\vec{x}_{i}\right)
$$

Thus, the average scattering rate for particles that result in energy deviation $|\delta|=\left|\frac{\Delta P}{P_{0}}\right|$ greater than a nominal value $\delta_{m}$ is given by

$$
\begin{aligned}
R_{\mathrm{MC}}\left(|\delta|>\delta_{m}\right) & =\frac{V}{N} \sum_{i=1}^{M}\left[\frac{v^{* \prime}}{\gamma^{2}} \frac{d \sigma^{* \prime}}{d \Omega^{* \prime}} \sin \Theta^{* \prime} \rho\left(\vec{x}_{1}\right) \rho\left(\vec{x}_{2}\right)\right]_{i} \\
& =\sum_{i=1}^{M} r_{i},
\end{aligned}
$$

where $N=2 \times N_{e}$ and $N_{e}$ is the total number of simulated scattering events (each event involves two particles); $V$ is the total volume in $\left(x, y, z, x_{1}^{\prime}, y_{1}^{\prime}, d p_{1}, x_{2}^{\prime}, y_{2}^{\prime}, d p_{2}, \Theta^{* \prime}\right.$, $\left.\Psi^{* \prime}\right)$, the 11-dimensional space from which the events are selected; $M$ is the total number of particles with energy deviation $|\delta|>\delta_{m}$ (one scattering event may generate one or two electrons with $|\delta|>\delta_{m}$, and they are counted individually), when $\delta_{m}$ equals the machine's momentum acceptance $\delta_{a}$, this equation gives the beam-loss rate; $r_{i}$ is the local scattering rate represented by each simulated scattered particles. When $N$ is large enough, the Monte Carlo integration converges to the analytical integral.

To use Monte Carlo integration, we need to generate a series of random scattering events. Each event involves a pair of scattering particles. The selection of the scattering particles, which we implemented in ELEGANT, is modified from Khan's work for BESSY II [10,11]. In ELEGANT, sample particles are selected from normalized phase space instead of from real phase space. In this way, we separated the particle's transverse motions into two parts, namely, a betatron oscillation and a dispersive orbit. As a result, the particle's density $\rho(\vec{x})$ in Eq. (8) can be written in a simple and standard format, making it easy to calculate correctly. In our method, the dispersion correction is done afterwards and the agreement between Piwinski's formula and our simulation results are good for all of the optical function regions (see Fig. 6). The original method combined the two parts of the motion when calculating $\rho(\vec{x})$ and gave larger simulation errors in dispersive regions. In ELEGANT, we also expanded the method to the case of general particle distributions rather than restricting it to a Gaussian beam. These two cases are discussed separately and in detail in below. 


\section{A. Monte Carlo simulation for a Gaussian-distributed bunch}

For a Gaussian-distributed bunch, nine uniformly distributed random numbers are generated in the normalized phase-space range from $[-r \sqrt{\varepsilon}, r \sqrt{\varepsilon}]$, where $r\left(r_{x}, r_{y}\right.$, or $\left.r_{z}\right)$ are factors supplied by the user, and $\varepsilon\left(\varepsilon_{x}, \varepsilon_{y}\right.$, or $\left.\varepsilon_{z}\right)$ are the beam emittances. These random numbers are assigned as: $\left(X_{1}, Y_{1}, X_{1}^{\prime}, Y_{1}^{\prime}\right)$ the normalized transverse coordinates of particle $1 ;\left(X_{2}^{\prime}, Y_{2}^{\prime}\right)$, the normalized transverse slopes of particle 2; $(s 1)$ the longitudinal coordinate of particle 1; and $\left(\frac{\Delta p_{1}}{p_{0}}, \frac{\Delta p_{2}}{p_{0}}\right)$ the fractional momentum deviations of particle 1 and 2 . The three spatial coordinates for particle 2 are identical to those for particle 1 , since the particles are colliding, so that $x_{2}=x_{1}=x, y_{2}=y_{1}=y$, and $s_{2}=$ $s_{1}=s$. Converting these coordinates to real phase space and including dispersion effect, particle 1 has

$z_{1}=\sqrt{\beta_{z}} Z_{1}+\frac{\Delta p_{1}}{p_{0}} D_{z}, \quad z_{1}^{\prime}=\frac{Z_{1}^{\prime}-\alpha_{z} Z_{1}}{\sqrt{\beta_{z}}}+\frac{\Delta p_{1}}{p_{0}} D_{z}^{\prime}$,

where $z(Z)$ represents $x(X)$ or $y(Y)$. For particle 2, we have

$$
\begin{aligned}
& Z_{2}=\left(z_{1}-\frac{\Delta p_{2}}{p_{0}} D_{z}\right) / \sqrt{\beta_{z}}, \\
& z_{2}^{\prime}=\frac{Z_{2}^{\prime}-\alpha_{z} Z_{2}}{\sqrt{\beta_{z}}}+\frac{\Delta p_{2}}{p_{0}} D_{z}^{\prime} .
\end{aligned}
$$

We now have a complete set of coordinates $\left(x, y, s, x_{1}^{\prime}, y_{1}^{\prime}, \Delta p_{1}, x_{2}^{\prime}, y_{2}^{\prime}, \Delta p_{2}\right)$ for both particles before scattering. The particle densities $\rho\left(\vec{x}_{1}\right)$ and $\rho\left(\vec{x}_{2}\right)$ in Eq. (8) can be easily calculated from the Gaussian function. The normalized phase-space volume is simply

$$
V_{n}=\left(2 r_{x} \sqrt{\varepsilon_{x}}\right)^{3}\left(2 r_{y} \sqrt{\varepsilon_{y}}\right)^{3}\left(2 r_{z} \sqrt{\varepsilon_{z}}\right)^{3} .
$$

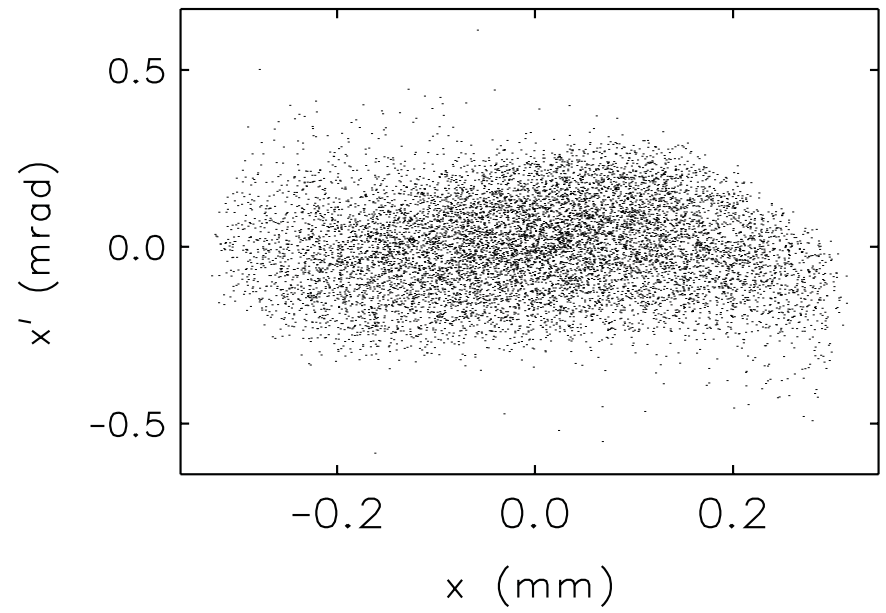

(a) horizontal
Transforming to real coordinates we have

$$
V_{p}=\frac{1}{\sqrt{\beta_{x} \beta_{y} \beta_{z}}} V_{n}
$$

To simulate the scattering process, another two random numbers are generated and assigned to $\Theta^{*}$ and $\Psi^{*}$. The scattered particle coordinates are obtained as shown in Fig. 1, and the volume corresponding to the range of scattering angles is $\pi^{2}$. The total 11-dimensional volume is $V=\pi^{2} V_{p}$. After choosing all the coordinates, the associated scattering rate in Eq. (8) for the event is calculated and stored for future use.

\section{B. Monte Carlo simulation for a general bunch}

A linac bunch is generally not Gaussian distributed. Figure 2 shows aspects of the particle distribution profile from an optimized high-brightness injector simulation for a possible APS-ERL upgrade [12]. Clearly, in this case, assuming a Gaussian-distributed bunch is a poor choice. On the other hand, modern computational capabilities make tracking billions of particles possible [13], which means that a detailed beam distribution function can be obtained. These facts push us to expand our Monte Carlo simulation to a general case-an arbitrarily distributed bunch.

To do this, first we need to know the particle distribution function at the location where we perform Monte Carlo simulation. In ELEGANT, this is done by tracking simulation particles through the beam line and making a multidimensional histogram of the particle distribution at the locations where scattering will be modeled. The number of bins in each dimension $\left(n_{x}, n_{y}, n_{s}, n_{x^{\prime}}, n_{y^{\prime}}, n_{\delta p}\right)$ is provided by the user.

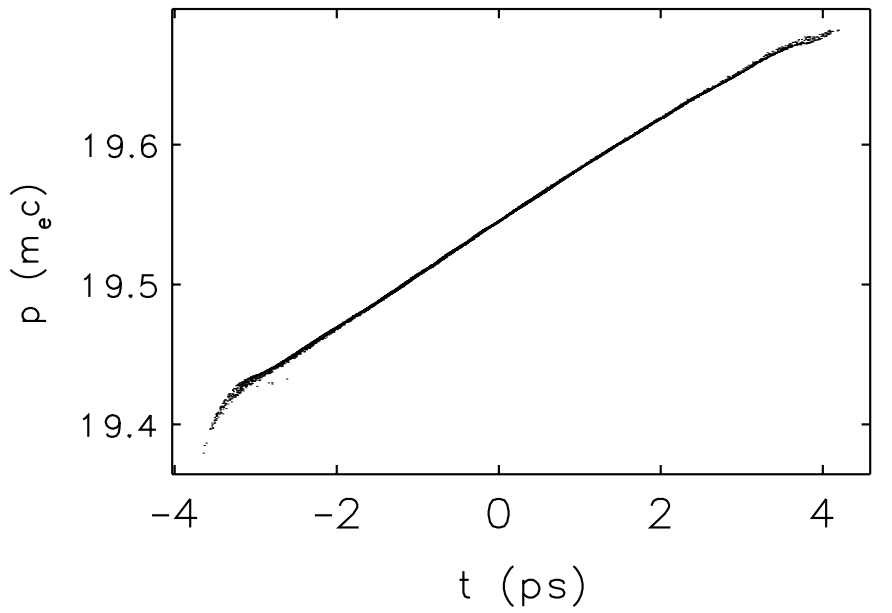

(b) longitudinal

FIG. 2. Particle distribution from optimized high-brightness injector simulation for a possible APS-ERL upgrade [12]. 
To be exact, a 6D histogram should be used to describe the particle distribution. However, this requires a very large number of particles in order to produce a detailed histogram. For example, to have 11 bins in each dimension, the total number of bins is $11^{6} \approx 2 \times 10^{6}$. The total number of particles needs to be significantly larger than this value. Another concern is that when interpolating a 6D table one must use the nearby $2^{6}=64$ grid point values. This is computationally expensive and may not always be necessary. To allow users to optimize the computations, ELEGANT supports three types of histogram input: full 6D histograms, or dual 4D (transverse) and 2D (longitudinal) histograms, or $2 \mathrm{D}$ histograms in each plane. In this paper, we use $4 \mathrm{D}+2 \mathrm{D}$ histograms to demonstrate our method. Figure 3 shows the histogram of a simulated electron bunch from a gun [12] which has 500000 particles. The index in the histogram is a $n \mathrm{D}$ subindex counter where each subindex is the bin index of the corresponding dimension. For example, the 4D counter is $\left[n_{x}\left|n_{x^{\prime}}\right| n_{y} \mid n_{y^{\prime}}\right]$, where $n_{x}$ is the bin index of $x$ and will take the value $\left[0, N B_{x}\right)\left(N B_{x}\right.$ is the total number of bins in the $x$ direction), and same for $n_{x^{\prime}}$, $n_{y}$, and $n_{y^{\prime}}$.

As described above, nine random numbers are generated within the ranges given by the histogram and used as the coordinates of the scattered particles' coordinates, $\vec{x}_{1}$ and $\vec{x}_{2}$. The values of $\rho\left(\vec{x}_{1}\right)$ and $\rho\left(\vec{x}_{2}\right)$ in Eq. (8) are computed from linear interpolation of the histogram values at the nearest grid points. The other aspects of the simulation are the same as the Gaussian-distributed bunch case.

\section{Selection of particles for tracking study}

Whether we use the Gaussian distribution or a distribution determined from tracking, we generate the same basic data, namely, the coordinates of the scattering particles and the particle density at their phase-space locations. Once we have these particles, the momenta are transformed to the c.m. system. The scattering angles $\Theta^{* \prime}$ and $\Psi^{* \prime}$ are, respectively, selected randomly within the regions $\left(0, \frac{\pi}{2}\right]$ and $[0,2 \pi]$ with uniform distributions. The momenta are rotated to the new position given by $\Theta^{* \prime}$ and $\Psi^{* \prime}$, and the corresponding Møller cross section is calculated. These are the momenta after scattering and are transformed back to the laboratory coordinate system and become $\left(p_{1}^{\prime}, p_{2}^{\prime}\right)$. The associated scattering probability rate $r_{i}$ is also calculated, using the local density values and the cross section.

At this point, we have the coordinates of the particles in the lab frame after scattering. If $\left|p_{1}^{\prime} / p_{0}-1\right|$ or $\mid p_{2}^{\prime} / p_{0}-$ $1 \mid$ is larger than the quantity $\delta_{m}$, which is specified by the user, then the particle and its associated scattering rate are saved for further use. Thus, the user-defined parameter $\delta_{m}$ specifies a minimum momentum deviation, below which losses are not expected in tracking. By repeating this process many times, the Monte Carlo integration of $R_{\mathrm{MC}}$ in Eq. (8) will reach a stable result and give an estimate of the scattering rate beyond the momentum deviation $\delta_{m}$.

Although ultimately we will use tracking to determine whether or not scattered particles are lost, we can also simply look at the scattering rate at any location as a function of the cutoff value $\delta_{m}$. This allows us to examine the convergence of Eq. (8). To do this we simulated up to $N=4 \times 10^{7}$ random events and computed the Touschek scattering rate $R_{\mathrm{MC}}$ for $\delta_{m}=0.001,0.01,0.03$, and 0.05 . Figure 4 shows $R_{\mathrm{MC}}$ (normalized to 1 for easy comparison) vs $N$ and $M$ at different $\delta_{m}$. It is clear that results converge for $N>2 \times 10^{7}$. For fixed $N, M$ varies in a large range for different $\delta_{m}$. When tracking, we normally want to know $M$ in advance, so we chose $M=5 \times 10^{6}$ as the default in ELEGANT. This number is only insufficient for small $\delta_{m}$, which can of course be changed by the user.

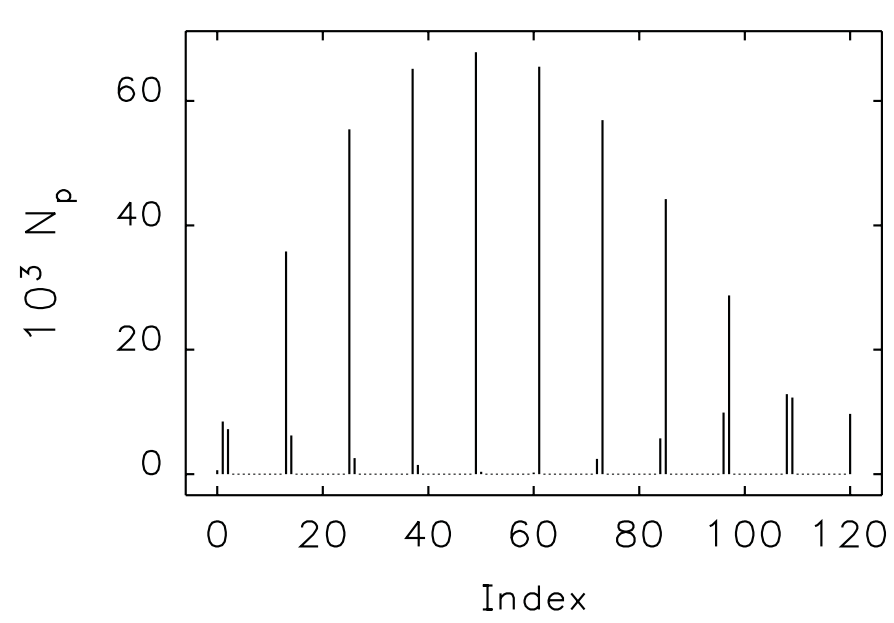

(a) longitudinal (2D)

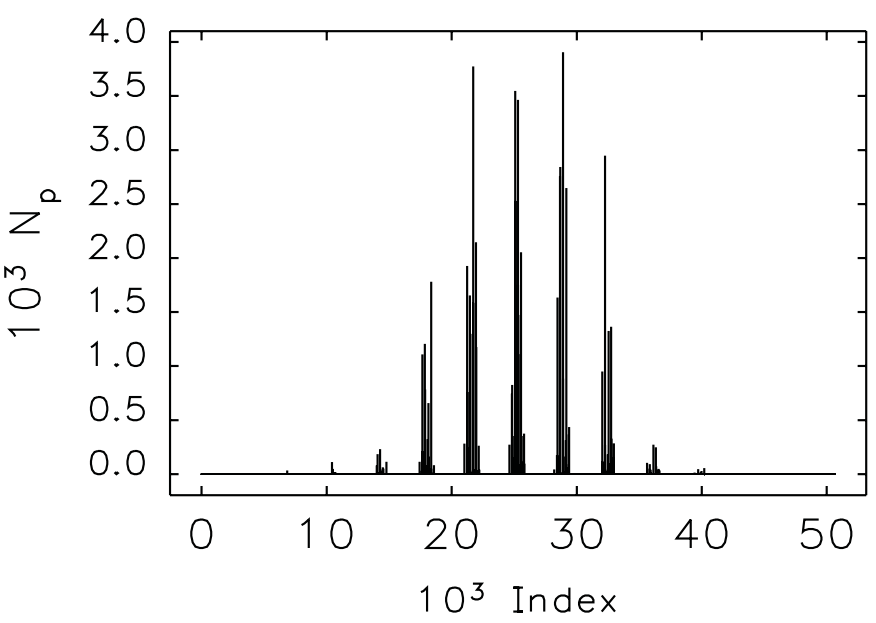

(b) transverse (4D)

FIG. 3. Histogram of a simulated bunch. 

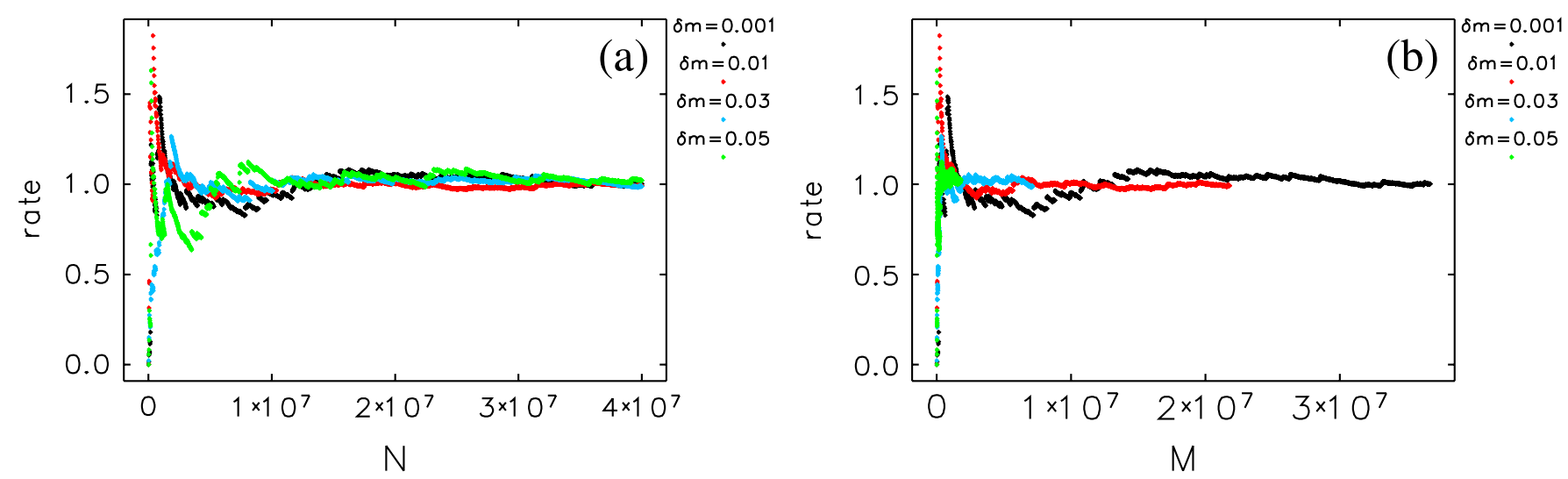

FIG. 4. (Color) Scattering rate (normalized to 1) vs number of simulated events $N$ (a) and number of satisfied scattering particles $M$ (b) at different $\delta_{m}$.

\section{Comparison of Monte Carlo simulation to Piwinski's formula}

To verify our scattering rate simulation, we applied both Piwinski's formula and Monte Carlo simulation to a turn-

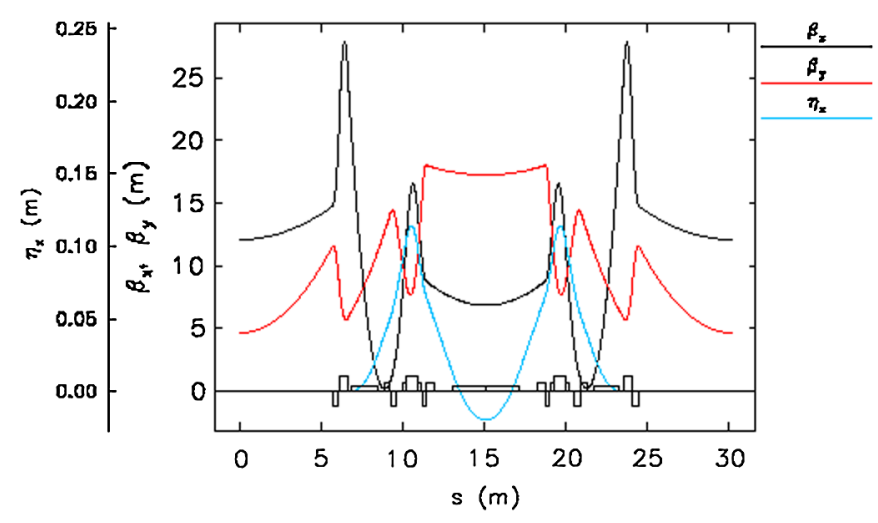

FIG. 5. (Color) Lattice used for simulation.

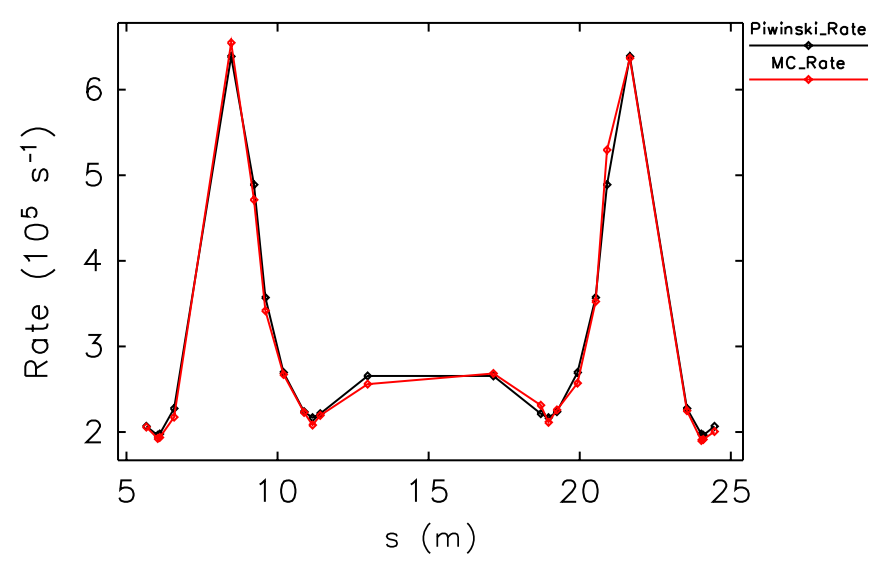

FIG. 6. (Color) Local Touschek scattering rate (1/s): Piwinski formula (black) and Monte Carlo simulation (red). around arc (TAA) cell of the APS-ERL lattice [8]. The optical functions of the TAA are illustrated in Fig. 5.

Figure 6 shows the local scattering rate calculated for $\delta_{m}=0.01$ for this $7 \mathrm{GeV}$ beam, which has $77 \mathrm{pC} /$ bunch, normalized transverse emittance $0.3 \mu \mathrm{m}$ in both planes, rms bunch length $0.6 \mathrm{~mm}$, rms energy spread $700 \mathrm{keV}$, and a repetition rate of $1.3 \mathrm{GHz}$. We see that the agreement is excellent.

Next, we computed the scattering rate as a function of beam energy and $\delta_{m}$. As Fig. 7 shows, the agreement is again excellent.

The comparison of calculated Touschek lifetime with experimental data has been made by many others [14-17]. Since our simulation code agrees well with the established Piwinski method, it should give the same level of agreement with experiment as in these papers.

ELEGANT also provides output of the simulated bunch particle distribution and the scattered particle distribution (histogram). These are shown in Figs. 8 and 9. The user can

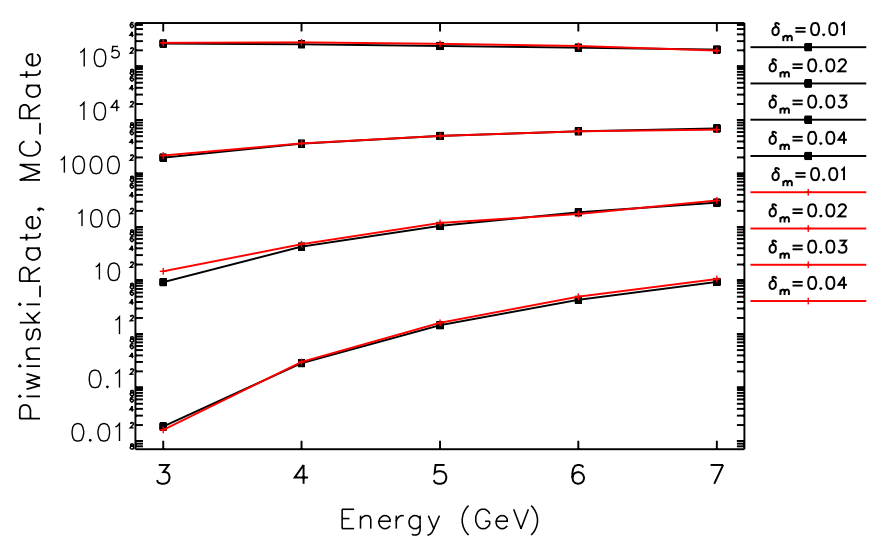

FIG. 7. (Color) The Touschek scattering rate $(1 / \mathrm{s})$ as a function of beam energy ( $x$ axis) and momentum acceptance $\delta_{m}$ (legend): Piwinski formula (black) and Monte Carlo simulation (red). 

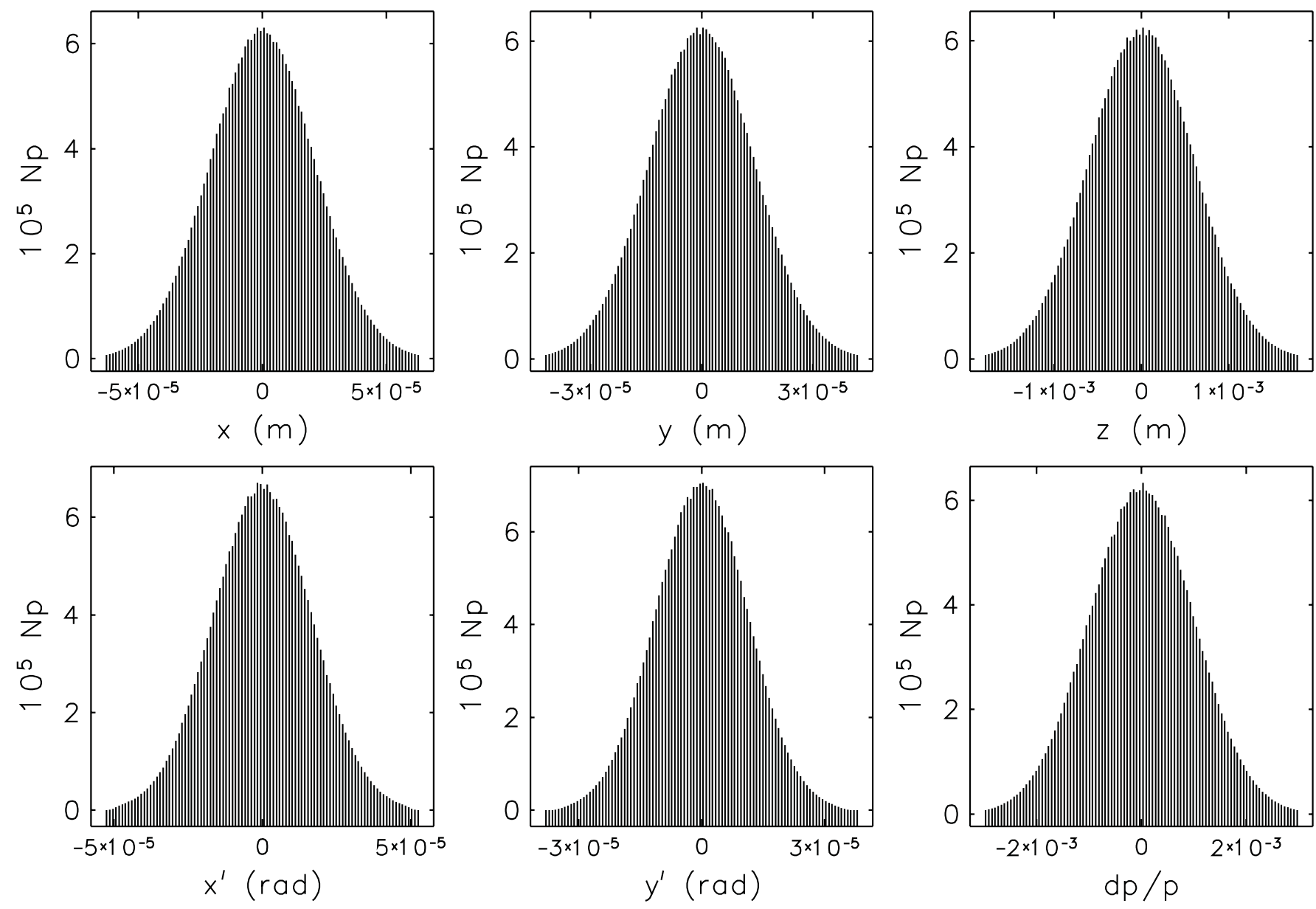

FIG. 8. Simulated bunch particle distribution.

check their simulation convergence by viewing these figures (the smoother the better).

\section{SIMULATION OF BEAM-LOSS RATE AND LOCATION}

Having validated our scattering rate simulation using Piwinski's formula, we are in a position to simulate beam loss due to Touschek scattering. The procedure is performed in several steps and illustrated in Fig. 10.

We used a lumped-element method to simulate beam loss due to Touschek scattering. The beam line under study is first divided into many small sections by inserting a special element TSCATTER at many locations. This is easily done using the ELEGANT command insertelements. The total number and locations where one should insert TSCATTER elements depends on the rapidity with which the energy and optical functions vary. To ensure reliable results, these variations should be small between successive scattering elements. The scattered particle distribution is obtained at each TSCATTER element using the Monte Carlo method described above.
The scattered particles are started at the location of the TSCATTER element. The scattering rate represented by each scattered particle is scaled with the total scattering rate integrated over the corresponding section (i.e., the section of the beam line between the current and previous TSCATTER element), see Eq. (13).

We wish to track scattered particles with $\delta>\delta_{m}$, where $\left(\delta_{m}\right)$ should be smaller than the local momentum acceptance $\delta_{a}$, in order to determine where they are lost. Unlike in storage rings, $\delta_{a}$ varies over a large range in linacs. Figure 11 shows the local momentum aperture determined by ELEGANT for the APS-ERL lattice. Similar results were reported for the Cornell ERL design [18]. In order to efficiently study beam-loss behavior from Touschek scattering, the local momentum aperture $\delta_{a, i}$ at each TSCATTER ( $i$ is the index of TSCATTER elements) is first calculated by using the momentum ${ }_{a}$ perture command, then results are scaled back to a slightly smaller value $\delta_{m, i}=a_{0} \delta_{a, i}$, where the user-controlled parameter $a_{0}\left(a_{0}<1\right.$ to ensure that we do not miss a possible lost particle) defaults to 0.85 . The correct value of $a_{0}$ depends on how momentum acceptance varies for particles with 

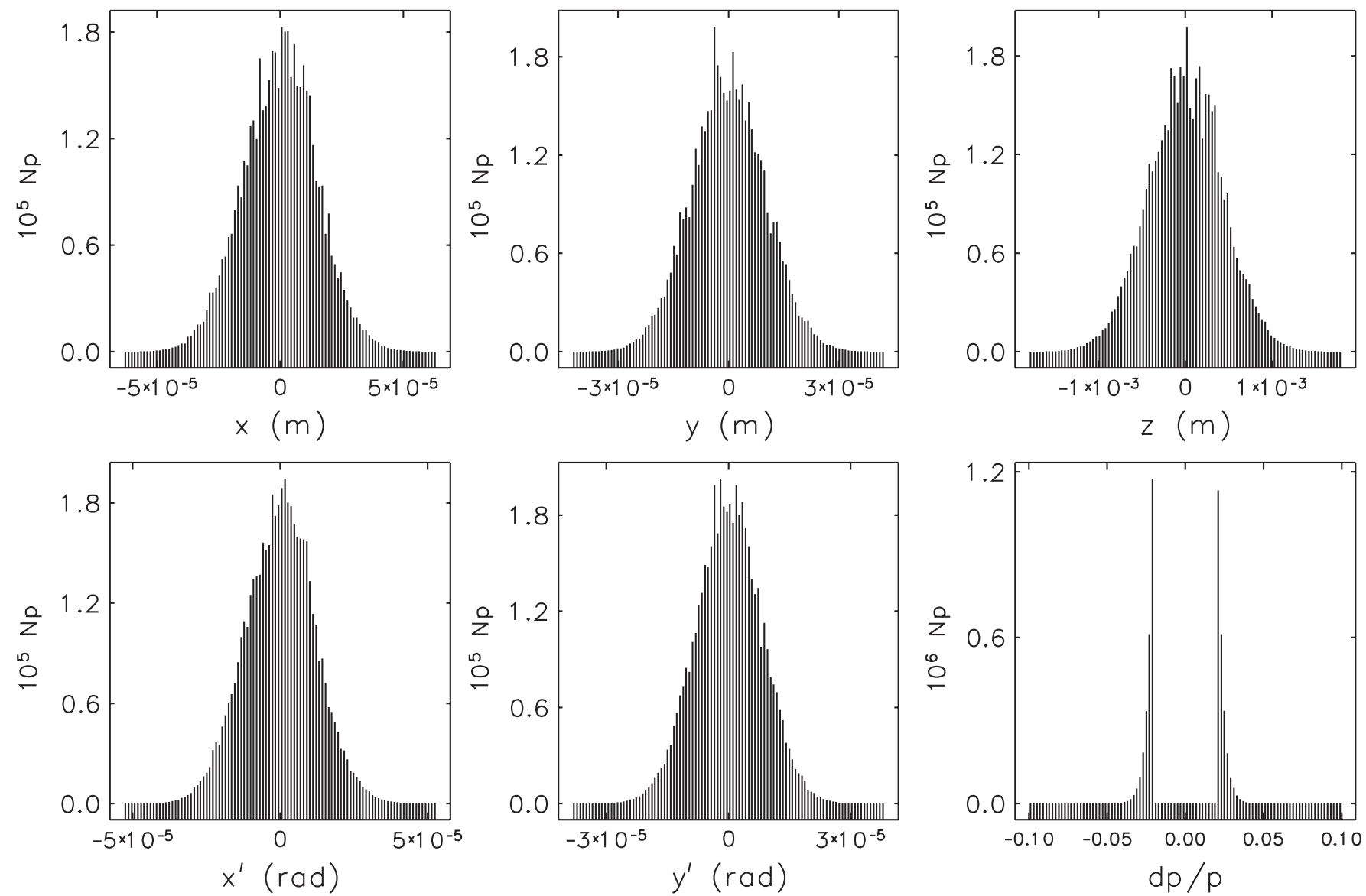

FIG. 9. Scattered particle distribution.

nonzero initial position and angle. Since it varies depending on the particular problem, it should be set with caution.

If the simulation is desired for a non-Gaussiandistributed bunch, then the particles distribution function

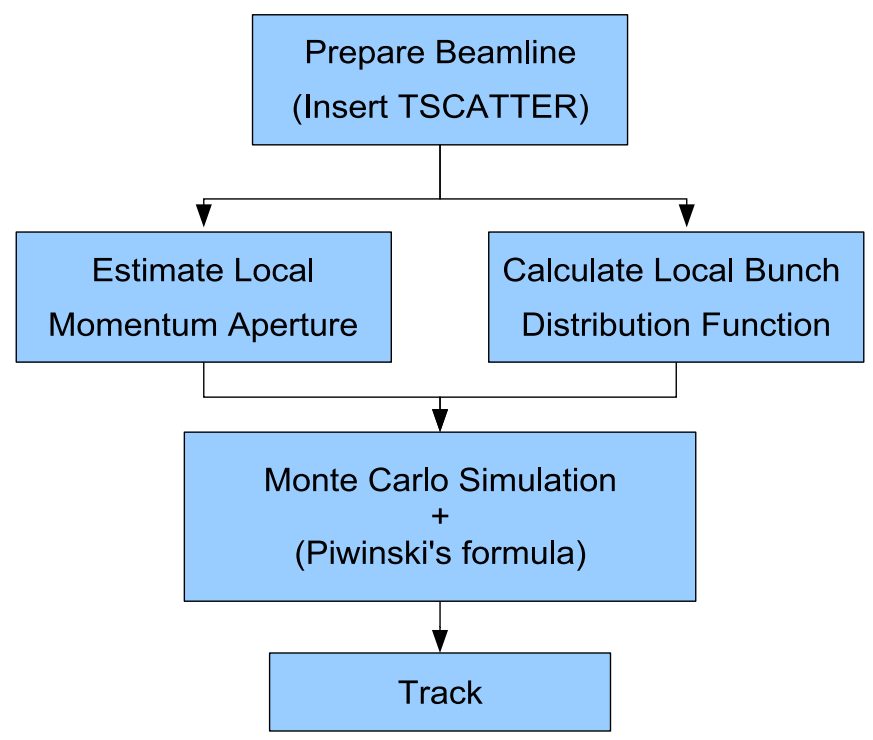

FIG. 10. (Color) Procedure of the Touschek-caused beam-loss simulation. at each TSCATTER needs to be known ahead of the simulation. This can be done by adding a MHISTOGRAM element at each TSCATTER position and then track the primary bunch through the beam line. The local bunch distribution function (histogram) is recorded at each MHISTOGRAM, and is used in the later Monte Carlo simu-

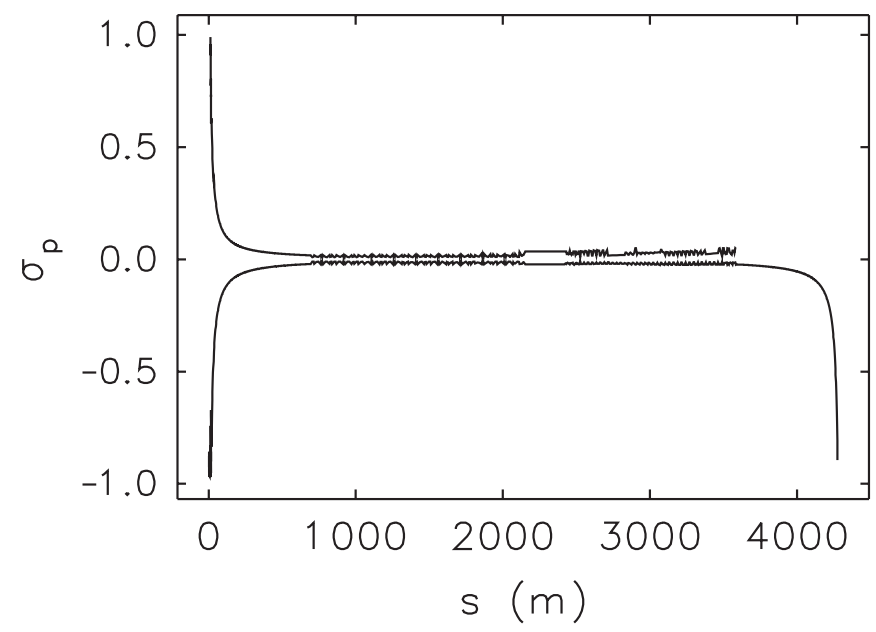

FIG. 11. Local momentum aperture for a possible APS-ERL lattice (tracking stopped at $E=189 \mathrm{MV}$ during deceleration). 
lation for generating scattered particles (secondary bunch) at each TSCATTER location. The reader can obtain more detailed information on the usage of MHISTOGRAM from [19].

Although the local scattering rate can be obtained using Monte Carlo, as we demonstrated above, it would be too time consuming to get an accurate value along an entire beam line section using this method. Piwinski's formula allows us to quickly determine the total scattering rate over each section for a Gaussian beam. This is used to weight the local simulation results at the TSCATTER element. Each simulated scattered particle having an associated local scattering rate $r_{i}$ given by Eq. (8) is then assigned an associated total scattering rate $R_{i}$ using

$$
R_{i}=\frac{r_{i}}{\sum r_{i}} \frac{R_{\mathrm{MC}}}{R_{\mathrm{P}}} \int R_{\mathrm{P}}
$$

where $R_{\mathrm{P}}$ and $R_{\mathrm{MC}}$ are local Touschek scattering rate from Piwinski's formula and Monte Carlo, respectively (for a Gaussian-distributed bunch, $\frac{R_{\mathrm{MC}}}{R_{\mathrm{P}}} \approx 1$ ). The integration is taken over the upstream section of the beam line.

In this way, the simulated scattered particles originating at the TSCATTER element accurately represent the scattering rate from the entire upstream section. The beam-loss rate and location can then be calculated through tracking those scattered particles through the beam line and recording all lost particles. The total loss rate will be the sum of $R_{i}$ for all the particles lost at any location.

\section{STRATEGY TO SPEED UP SIMULATION}

As we pointed out earlier, to obtain a reliable scattering rate and a smooth scattered electron distribution, $M$ has to be a large number $\left(M \sim 5 \times 10^{6}\right)$. In addition, a large number of beam line sections, $N_{s}$, is also required. That means that we need to track $M \times N_{s}$ electrons, which requires significant CPU time. This motivated development of a strategy to reduce the simulation burden. We examined the scattering rate that each simulated electron represents and found, not surprisingly, a large variation. Some simulated electrons represent highly probable scattering events, while some represent very improbable events.

To illustrate this, we took the data from our previous simulation and sorted the simulated electrons in order of increasing associated scattering rate. Figure 12 illustrates the accumulation of the scattering rate $\left(\sum r_{i}\right)$ vs the number of simulated electrons $(M)$.

From this plot we can see that about $18 \%$ of the simulated electrons represent about $99.9 \%$ of the scattering rate, which means that we may track a small portion of the simulated electrons and get good beam-loss information. Figure 13 compares the computed loss rate distributions for tracking scattered electrons with $95 \%, 99 \%$, and $100 \%$ of the total scattering rate, respectively. It is evident that the differences are small and that this strategy can greatly

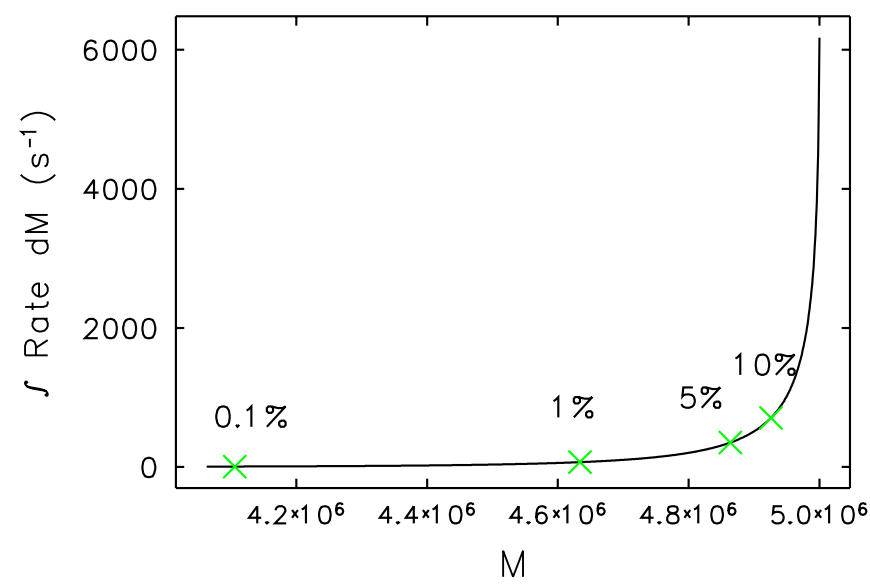

FIG. 12. (Color) Integrated scattering rate vs number of simulated electrons. Electrons are sorted with increasing associated scattering rate.

speed up simulation with little sacrifice of accuracy. In ELEGANT we use $99 \%$ as the default value; that is, by default we only track the highest-weight particles that represent $99 \%$ of the scattering rate.

Most likely, the particles dropped from tracking are those with large momentum deviation, which will be lost very soon after scattering. It may not always be desirable to ignore these particles. The error in doing so depends on tracking results. For example, if we track particles representing $99 \%$ of the scattering rate and the losses are $50 \%$ of the total scattering rate, then the total loss is very likely to be $51 \%$. The relative error by dropping those low weight particles will be $\sim \frac{1}{50}$. Hence, it is advisable to choose $\delta_{m}$ as close as possible to the momentum aperture (in this case, most particles will be lost somewhere), and vary the cutoff level to ensure correct results.

\section{APPLICATION TO APS-ERL DESIGN}

APS is exploring a possible ERL upgrade, but we are concerned about beam-loss issues in the present APS tunnel. The allowed beam loss in the APS ring is about $170 \mathrm{pA} / 1100 \mathrm{~m}$ [20], i.e., $0.15 \mathrm{pA} / \mathrm{m}$, to be compared to an average current of 25 to $100 \mathrm{~mA}$. We applied the method described above to the APS-ERL lattice [8]. The simulated beam was from an optimized high-brightness injector simulation [12]: it has the normalized geometric rms emittance of $0.35 \mu \mathrm{m}, 33.5 \mathrm{keV} \mathrm{rms}$ energy spread at $12.5 \mathrm{MeV}, 0.55 \mathrm{~mm}$ rms bunch length, $77 \mathrm{pC}$ charge per bunch, and a 1.3-GHz repetition rate, giving 100-mA average current. Figure 14(a) shows the beam-loss rate from Touschek scattering without sextupole correction in the TAA section. This is well above what is tolerable.

A sextupole correction along the TAA section was then added to maximize the local momentum aperture. To do this, the local momentum aperture is first computed by tracking particles from each location (e.g., the end of each 

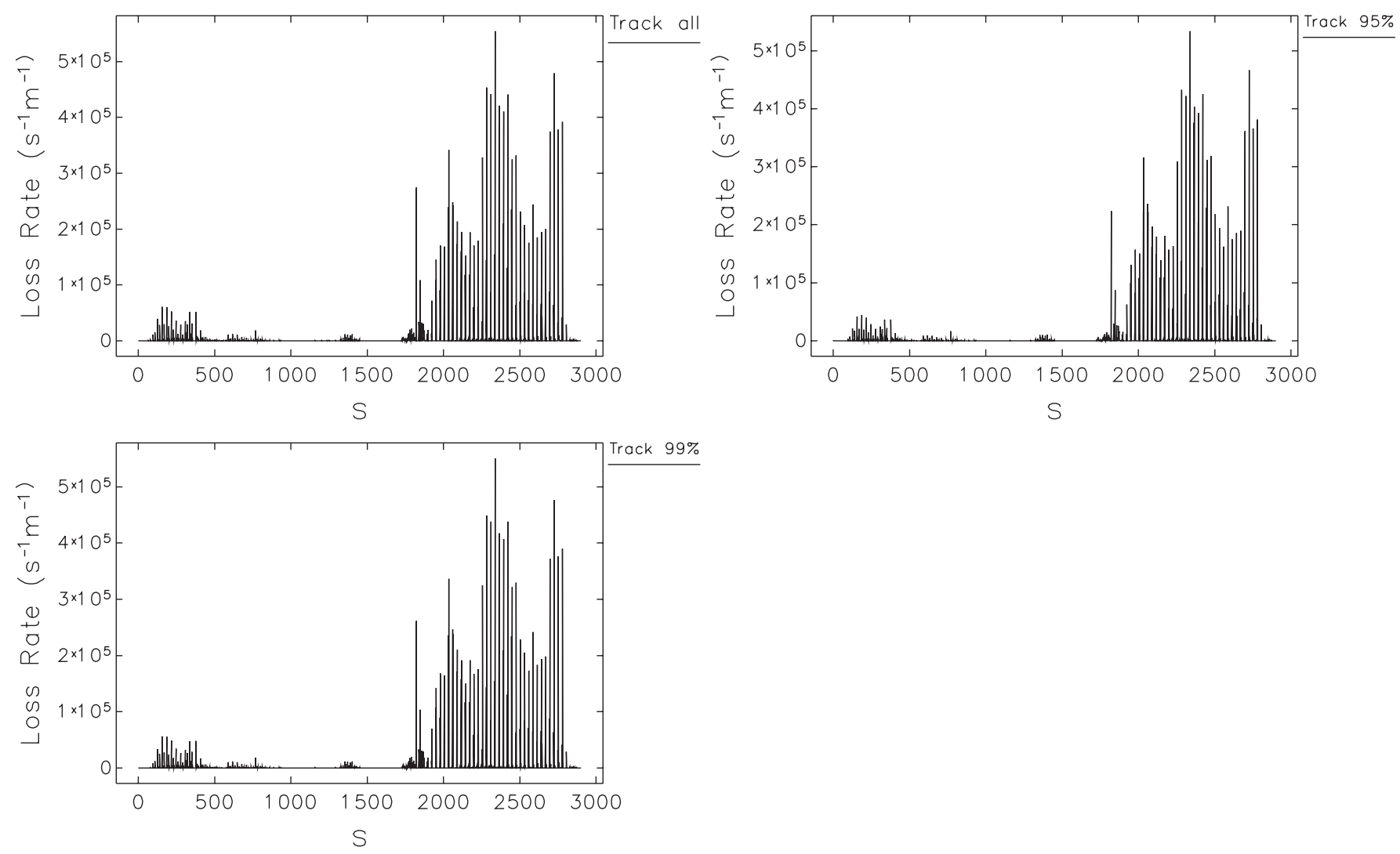

FIG. 13. Simulated loss rate vs position for various values of the scattering rate cutoff ( $s$ starts from the end of linac; APS ring starts at $s \approx 1900 \mathrm{~m})$.

beam line element) with increasing positive and negative momentum deviations, until a loss is seen. We find the minimum absolute value of the momentum deviation limit for all starting locations, then maximize this value by adjusting sextupole families. Figure 14(b) shows that this optimization significantly reduces the beam-loss rate; the average loss rate $(0.018 \mathrm{pA} / \mathrm{m})$ is well below the desired value.
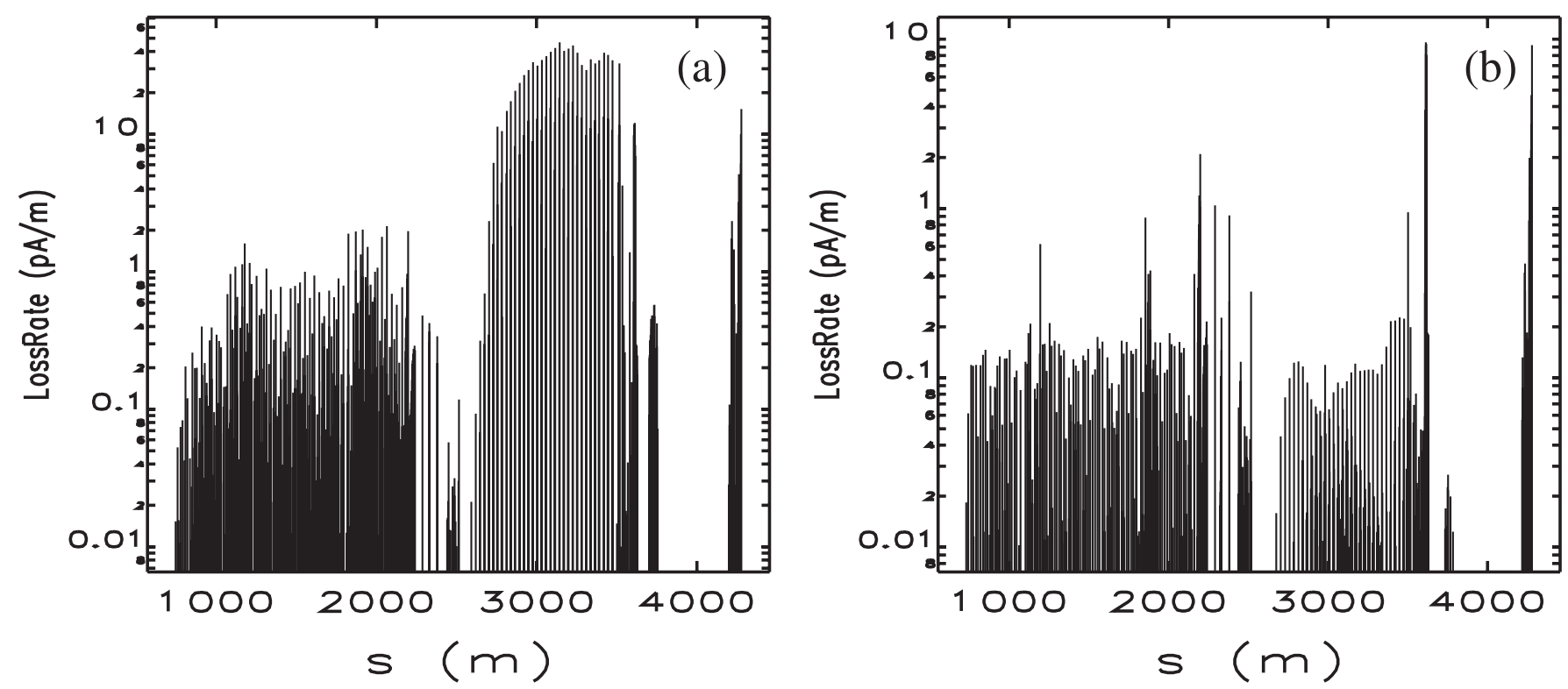

FIG. 14. Simulated loss rate vs position for APS-ERL: (a) without sextupole optimization; (b) with optimized sextupoles ( $s$ starts from the end of electron gun; APS starts at $s \approx 2600 \mathrm{~m}$ ). 
The user also can obtain beam profile information by dumping surviving scattered particles using the WATCH element provided by ELEGANT. In this case, the input $\delta_{m}$ should be significantly smaller than the momentum aperture limitation. This information can be used for later collimator system design [21].

\section{SUMMARY}

A Monte Carlo simulation of Touschek scattering for a single-pass system (linac or transport line) was implemented in ELEGANT. We compared the simulated local scattering rate with the rate from Piwinski's formula for a Gaussian-distributed bunch and found good agreement, which confirms that our scattering simulation is correct. We extended the simulation to allow an arbitrarily distributed bunch. This involves first tracking with a simulated bunch to create histograms describing the bunch distribution, then using these histograms to compute the scattering rates. We also developed a strategy to speed up the tracking simulation by selecting only those simulated scattered particles that represent the bulk of the scattering rate. Application to a proposed APS-ERL lattice shows that the Touschek scattering effect is serious for such a highbrightness electron beam. An optimized sextupole correction must be employed to lower the beam loss to a safe value.

\section{ACKNOWLEDGMENTS}

We would like to thank Professor S. Khan for providing his code and Dr. Louis Emery for many useful discussions. This work was supported by the U.S. Department of Energy, Office of Science, Office of Basic Energy Sciences, under Contract No. DE-AC02-06CH11357.

[1] C. Bernardini, G. F. Corazza, G. Di Giugno, G. Ghigo, J. Haissinski, P. Marin, R. Querzoli, and B. Touschek, Phys. Rev. Lett. 10, 407 (1963).

[2] H. Bruck, Los Alamos Report No. LA-TR-72-10 Rev, 1965, English translation.
[3] A. Piwinski, DESY Report No. DESY 98-179, 1998.

[4] M. Tigner, Nuovo Cimento 37, 1228 (1965).

[5] M. Borland et al., Nucl. Instrum. Methods Phys. Res., Sect. A 582, 54 (2007).

[6] A. Xiao and M. Borland, in Proceedings of the 2007 Particle Accelerator Conference, Albuquerque, New Mexico (IEEE, Albuquerque, New Mexico, 2007), p. 3453.

[7] M. Borland, Advanced Photon Source LS-287, 2000.

[8] M. Borland et al., in Proceedings of the Eighth International Topical Meeting on Nuclear Applications and Utilization of Accelerators (AccApp'07), Pocatello, Idaho (American Nuclear Society, LaGrange Park, Illinois, 2007), p. 196.

[9] W. T. Ford, A. K. Mann, and T. Y. Ling, Report No. SLAC158, 1972.

[10] S. Khan, in Proceedings of the EPAC 94, London, U.K. (World Scientific Publishing, Singapore, 1994), p. 1192.

[11] S. Khan (private communication).

[12] X. Dong (private communication).

[13] J. Qiang, in Proceedings of ICAP09, San Francisco, USA.

[14] M. Takao, K. Soutome, and H. Tanaka, in Proceedings of the European Particle Accelerator Conference, Vienna, 2000 (EPS, Geneva, 2000), pp. 1569-1571.

[15] N.P. Abreu and P.F. Tavares, in Proceedings of the 9th European Particle Accelerator Conference, Lucerne, 2004 (EPS-AG, Lucerne, 2004), pp. 2266-2268.

[16] A. Mochihashi et al., in Proceedings of the 8th European Workshop on Beam Diagnostics and Instrumentation for Partical Accelerators, 2007 (Venice, 2007), p. 280 [http:// www.JACoW.org].

[17] L. Yang, B. Podobedov, S. L. Kramer, and S. Y. Lee, in Proceedings of the 2007 Particle Accelerator Conference, Albuquerque, New Mexico (Ref. [6]), pp. 1203-1205.

[18] G. Hoffstaetter, M. P. Ehrlichman, and A. Temnykh, in Proceedings of the 11th European Particle Accelerator Conference, Genoa, 2008 (EPS-AG, Genoa, Italy, 2008), p. 1631 [www.JACoW.org].

[19] User's Manual for ELEGANT.

[20] "Advanced Photon Source Safety Assessment Document," APS-3.1.2.1.0 Rev. 3, 2006.

[21] A. Temnykh, M.P. Ehrlichman, and G. Hoffstaetter, in Proceedings of the 11th European Particle Accelerator Conference, Genoa, 2008 (Ref. [18]), p. 214 [www.JACoW.org]. 